\title{
The gridded retarding ion drift sensor for the petitsat cubeSat mission
}

Cite as: Rev. Sci. Instrum. 91, 064502 (2020); https://doi.org/10.1063/1.5140470

Submitted: 27 November 2019 . Accepted: 20 May 2020 . Published Online: 17 June 2020

R. L. Davidson (D), B. Oborn, E. F. Robertson (D), S. Noel, C. D. Earle (D), J. Green, and J. Kramer
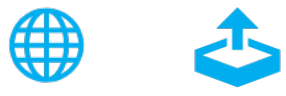

\section{ARTICLES YOU MAY BE INTERESTED IN}

Ion temperature measurement techniques using fast sweeping retarding field analyzer (RFA) in strongly intermittent ASDEX Upgrade tokamak plasmas

Review of Scientific Instruments 91, 063506 (2020); https://doi.org/10.1063/5.0010788

A high-resolution spectroscopic system for remote measurement of plasma parameters Review of Scientific Instruments 91, 063502 (2020); https://doi.org/10.1063/1.5141486

A pulsed-power implementation of "Laser Gate" for increasing laser energy coupling and fusion yield in magnetized liner inertial fusion (MagLIF)

Review of Scientific Instruments 91, 063507 (2020); https://doi.org/10.1063/1.5139663

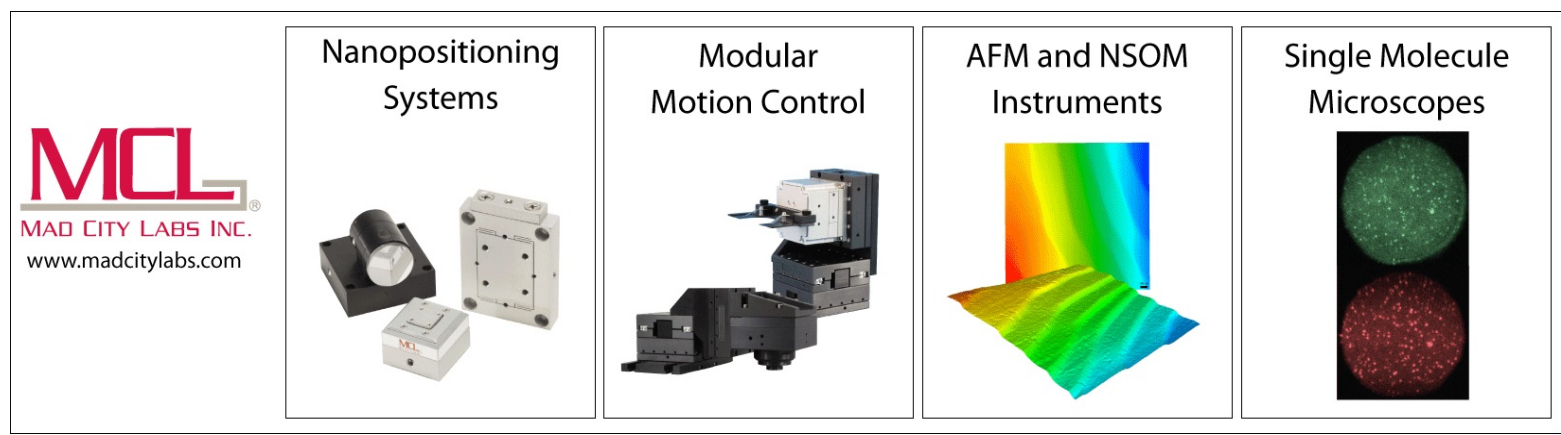




\title{
The gridded retarding ion drift sensor for the petitSat cubeSat mission
}

\author{
Cite as: Rev. Sci. Instrum. 91, 064502 (2020); doi: 10.1063/1.5140470 \\ Submitted: 27 November 2019 • Accepted: 20 May 2020 • \\ Published Online: 17 June 2020
}

\author{
R. L. Davidson, ${ }^{1, a)}$ (iD B. Oborn, ${ }^{2}$ E. F. Robertson, ${ }^{2}$ (D) S. Noel, ${ }^{2}$ G. D. Earle, ${ }^{2}$ (D) J. Green, ${ }^{2}$ and J. Kramer
}

\begin{abstract}
AFFILIATIONS
${ }^{1}$ Electrical and Computer Engineering, Utah State University, Logan, Utah 84322, USA

${ }^{2}$ Electrical and Computer Engineering, Virginia Tech, Blacksburg, Virginia 24061, USA
\end{abstract}

a) Author to whom correspondence should be addressed: ryan.davidson@usu.edu

\begin{abstract}
The Gridded Retarding Ion Drift Sensor (GRIDS) is a small sensor that will fly on the $6 \mathrm{U}$ petitSat CubeSat. It is designed to measure the three-dimensional plasma drift velocity vector in the Earth's ionosphere. The GRIDS also supplies information about the ion temperature, ion density, and the ratio of light to heavy ions present in the ionospheric plasma. It utilizes well-proven techniques that have been successfully validated by similar instruments on larger satellite missions while meeting CubeSat-compatible requirements for low mass, size, and power consumption. GRIDS performs the functions of a Retarding Potential Analyzer (RPA) and an Ion Drift Meter (IDM) by combining the features of both types of instruments in a single package. The sensor alternates RPA and IDM measurements to produce the full set of measurement parameters listed above. On the petitSat mission, GRIDS will help identify and characterize a phenomenon known as plasma blobs (or enhancements).
\end{abstract}

Published under license by AIP Publishing. https://doi.org/10.1063/1.5140470

\section{INTRODUCTION}

The petitSat CubeSat mission is an upcoming NASA-funded $6 \mathrm{U}$ CubeSat mission focused on studying the link between plasma enhancements in the ionosphere and medium-scale traveling ionospheric disturbances (MSTIDs). ${ }^{1}$ These two phenomena have various characteristics that manifest as perturbations to the background ionospheric conditions as well as closely related background conditions in the thermosphere. For that reason, the petitSat mission will rely on two main science sensors: one to measure the plasma in the ionosphere and the other to measure the neutral gas in the thermosphere. This paper focuses on describing the functioning of the instrument tasked with quantifying changes in the ionosphere. Details of the mission are not the focus of this paper, but they will be presented in other publications.

Retarding potential analyzers have a long history of providing in situ diagnostics of the thermal ion distribution in the Earth's ionosphere. The practical and theoretical underpinnings of the technique are well described in the literature, ${ }^{2}$ and retarding potential analyzer (RPA) instruments have successfully flown on many large satellite missions, including Dynamics Explorer (DE), ${ }^{3}$ Atmosphere Explorer (AE), ${ }^{4}$ and the defense meteorological satellite program
(DMSP) series. ${ }^{5}$ Reasons for the popularity of RPAs include their relative simplicity, high reliability, and the large number of state variables that can be inferred from their measurements. When coupled with good spacecraft velocity data and attitude knowledge, analysis of the current-voltage characteristics produced by an RPA provides measurements of the overall plasma density, the ion temperature, the component of the ion velocity vector along the orbit-track, and the ratio of light ions $\left(\mathrm{H}^{+}, \mathrm{He}^{+}\right)$to heavy ions $\left(\mathrm{O}^{+}, \mathrm{NO}^{+}\right.$, etc.) in planetary ionospheres. Very few single-sensor spacecraft instruments simultaneously measure such a large number of geophysically significant parameters.

The limitation of the RPA in characterizing the full drift vector has frequently led to the obvious pairing of an RPA with a complementary instrument designed to measure the remaining two components of the ion drift velocity vector. Often referred to as an Ion Drift Meter (IDM), ${ }^{6}$ this instrument has been utilized along with an RPA on all of the high-profile missions listed above, as well as more modern missions such as ICON. ${ }^{7}$ While this pairing offers a comprehensive and robust approach to in situ thermal ion diagnostics, it is often too large to be utilized on CubeSats or other small satellites. The Gridded Retarding Ion Drift Sensor (GRIDS) instrument combines the functionality of the RPA and IDM into one sensor-head 
and is specifically designed for compatibility with the size, mass, and power restrictions imposed by CubeSat platforms.

GRIDS shares many similarities with both the RPA and the IDM. The sensor head $(9.5 \mathrm{~cm}$ square $)$ consists of a square aperture that is mounted on the ram face $(10 \times 30 \mathrm{~cm}$ side $)$ of a CubeSat. The goal is to make the aperture as small as possible and to reduce the impact of non-uniform sheath effects while maintaining an adequate signal to noise ratio (SNR) to ensure good performance in the ionospheric regions of interest. The GRIDS sensor is shown in Fig. 1.

Behind the aperture is a series of biased and grounded grids that act as ion-energy filters. GRIDS has two distinct measurement modes. When the instrument is in RPA mode, one of its internal grids is swept over a range of voltages so it functions like the retarding grid in an RPA. In IDM mode, all the grid voltages are held constant, and one grid is held at a static potential sufficient to screen out light ions. Behind the grid stack are four segmented collector elements at the back of the instrument. As ions impact these segments, they produce currents to each segment that are separately measured by the GRIDS electronics. In RPA mode, the current from the four segments is added together to allow for the calculation of the total ion flux as a function of retarding voltage, which is related to the ion density, temperature, relative concentration of heavy to light ions, and the component of the velocity vector normal to the instrument face. In IDM mode, the ratios of the current collected by pairs of collector segments are calculated. When combined with knowledge of the instrument dimensions, spacecraft attitude, and grid configuration, these ratios allow the arrival angle of the incoming ion beam to be calculated in the frame of reference of the spacecraft. This angle can be used in conjunction with the ram velocity component determined in RPA mode, thereby providing a complete determination of the three-dimensional ion drift velocity vector over the desired range of $-500 \mathrm{~m} / \mathrm{s}$ to $500 \mathrm{~m} / \mathrm{s}$ in each component.

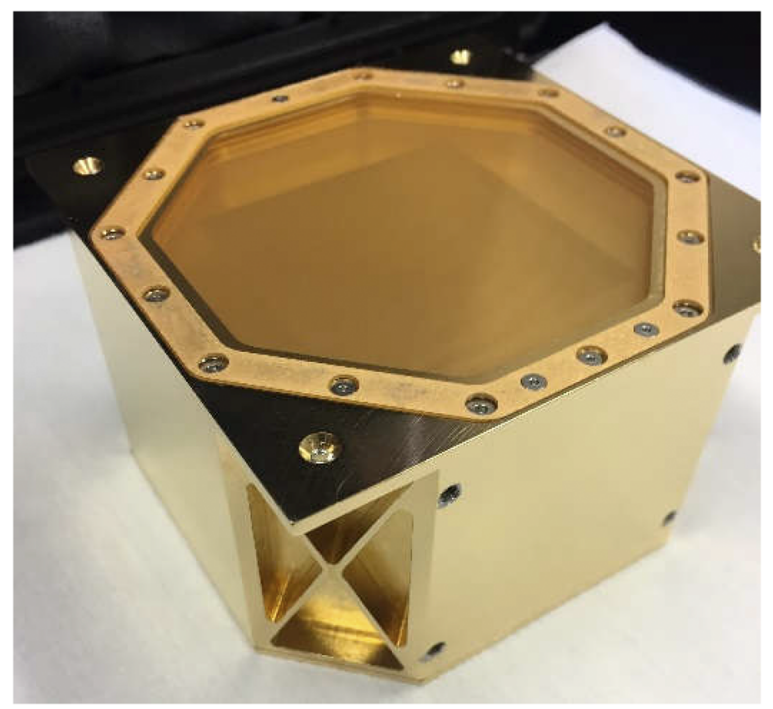

FIG. 1. The GRIDS sensor.

\section{RPA MEASUREMENT}

The Retarding Potential Analyzer (RPA) has a long history of use in ionospheric studies. It has been proven as a robust and versatile instrument that returns a relatively large amount of data related to the plasma conditions in the ionosphere. The technique has been described repeatedly and extensively in the literature. ${ }^{8-12}$ GRIDS utilizes a double-retarding grid design with a retarding voltage that is swept from 0 to $12 \mathrm{~V}$ over 40 discrete steps.

\section{IDM MEASUREMENT}

The Ion Drift Meter (IDM) has been the companion instrument to the retarding potential analyzer on numerous high profile missions, including Atmospheric Explorer, Dynamics Explorer, the Defense Meteorological Satellite Program, and the Communication/Navigation Outage Forecast System. Together, these two instruments allow for the determination of many of the most important properties of the thermalized ionospheric plasma. The RPA is responsible for determining the composition, density, temperature, and ram component of the velocity vector of a drifting Maxwellian plasma, while the IDM measures the other two orthogonal components of the velocity vector.

The IDM shares many similarities with the RPA in that it consists of an aperture, numerous biased grids, and a solid plate collector. This instrument has been described in detail by Heelis and Hanson. ${ }^{6}$ When mounted on a satellite, the IDM consists of a grounded grid exposed to the plasma and facing in the ram direction, followed by a grid with a small positive potential to reject light ions and a square collimating aperture. Beyond the collimator, there is an electric field-free region known as the "drift region." Finally, there is a suppressor grid held at a large negative potential (usually $-12 \mathrm{~V}$ ) to reject ambient electrons and turn back secondary and photoelectrons produced at the solid plate collector by ions and photons incident on the conductive collector surfaces. A cross section view of the IDM is shown in Fig. 2. The collector is segmented into four squares that are aligned so that their separation lines are parallel to the edges of the square collimator. Incident ions are formed into a beam with a square cross section by the collimator. The square

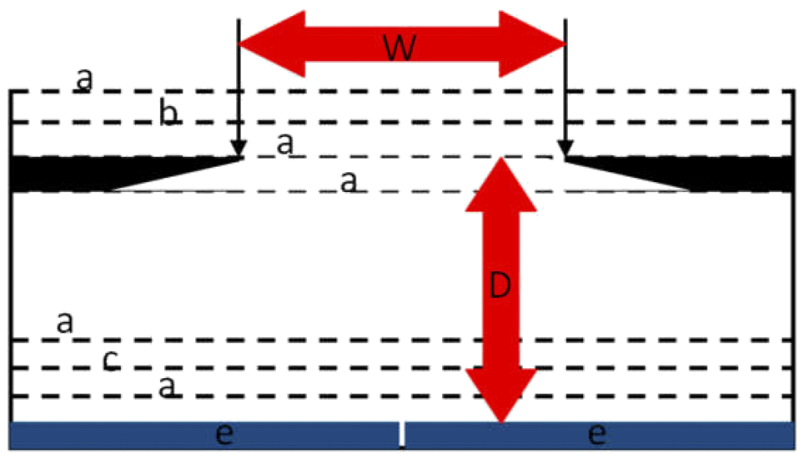

FIG. 2. A conceptual cross section of an ion drift meter. Grounded grids are marked as $a, b$ is the light ion rejection grid, and $c$ is an electron suppression grid. $W$ is the width of the aperture and $D$ is the distance between the aperture and the collectors. 
beam passes through the drift region at an angle of incidence determined by the ratio of the ram to cross-track speeds. By the time the beam reaches the four collectors, it has shifted in space relative to the square aperture. The beam illuminates a square region somewhere on the four segments of the collector. The current collected by each segment is proportional to the illuminated area on that segment. The current to all four collector segments is compared, allowing the amount the beam has shifted by to be calculated. The width of the square aperture and the length of the drift region are well known, so the angle of the incoming beam relative to the normal to the instrument aperture plane can be obtained in two dimensions. With the angle of the incoming beam and knowledge of the velocity of the plasma in the ram direction, the other two orthogonal components of the velocity vector can be calculated.

The geometry underlying the measurement of the arrival angles enters into the relationship between the collected current and the angles in a straightforward manner. The current to either half of the collector (two adjacent segments) can be written as

$$
I=q N A v \chi,
$$

where $\mathrm{q}$ is the charge of the incoming ions, $\mathrm{N}$ is the ion density, $\mathrm{A}$ is the area illuminated by the incoming beam, $v$ is the beam velocity, and $\chi$ is the transparency of the grid stack. Taking the ratio of the current to the two collector halves, the following expression is arrived at:

$$
R=\frac{I_{1}}{I_{2}}=\frac{\frac{w}{2}+D \tan (\theta)}{\frac{w}{2}-D \tan (\theta)},
$$

where $\mathrm{W}$ and $\mathrm{D}$ are defined in Fig. 2, $\mathrm{R}$ is the current ratio, and $\theta$ is the angle of the incoming ion beam with respect to the ram direction and orthogonal to the dividing line between the two collectors used. Equation (2) can then be solved for $\theta$ to yield

$$
\theta=\tan ^{-1}\left[\frac{W(1-R)}{2 D(1+R)}\right] .
$$

For the small angles associated with the cross-track to ram speed ratios, this equation is nearly linear. Equation (3) can be used to measure the angle of the incoming ion beam by simply using the ratio of the measured currents to each half of the instrument. With the knowledge of the ram velocity of the ions (the spacecraft velocity can be used as a first-order approximation), the cross-track velocity in one direction can be obtained. This procedure is carried out for both sets of opposing halves of the collector to determine both components of the velocity vector in the plane of the instrument aperture.

The above calculation for the angle would be correct except for the fact that, like a beam of light passing through materials with different indices of refraction, a beam of ions does not maintain a linear path with a single slope as it passes through areas with different electric field strengths. There are two grids held at constant bias potentials within the instrument: the hydrogen repelling grid and the suppressor grid. The positive hydrogen repeller will slow the beam and thus deflect it away from the normal, while the negative suppressor will deflect the beam toward the normal. Since the positive repeller grid is encountered by the incident ions before the collimator and the beam is returned to its initial angle by the time it reaches the second grounded grid, this displacement has no effect. The suppressor grid, however, is between the collimator and the collector, and thus, the displacement will lead to an altered current ratio, which leads to an error in the angle. The displacement can be accounted for by determining the time it takes for the beam to traverse the gap between the two grounded grids surrounding the suppressor and then modifying D in Eq. (3). It can be easily seen that the acceleration between a grounded grid and the suppressor is

$$
a=\frac{q \phi}{m s}
$$

where $\mathrm{q}$ is the charge on the ions, $\varphi$ is the suppressor potential, $\mathrm{m}$ is the mass of the ions, and $\mathrm{s}$ is the spacing between the grids. Given this acceleration, the time it takes the ions to traverse the distance $s$ is

$$
t=\frac{-v_{0}+\sqrt{v_{0}^{2}-2 s a}}{a},
$$

where $\mathrm{v}_{0}$ is the initial velocity of the beam in the ram direction. Using this time, an equivalent distance can be calculated assuming that the beam remained at its initial velocity,

$$
s^{\prime}=v_{0} t=v_{0}\left(\frac{-v_{0}+\sqrt{v_{0}^{2}-2 s a}}{a}\right) .
$$

Note that this reduced distance works for both the distance between the first grounded grid and the suppressor and the distance between the suppressor and the collector. Equation (6) can be used to form a corrected drift distance,

$$
D^{\prime}=D-2 s+2 s^{\prime}
$$

The reduced drift distance in Eq. (7) can be used in Eq. (3) to correct for the displacement of the beam as it passes through the suppressor.

One final correction is required to account for spacecraft charging. Depending on the ion and electron collection areas on the ram, wake, and sides of the spacecraft, it is possible for the satellite to collect more electrons than ions as it orbits the Earth. This asymmetric charge collection leads to the spacecraft retaining some small negative potential (usually a volt or two) relative to the plasma potential. Ions that enter the instrument begin at the plasma potential but are accelerated through the sheath and gain energy if the spacecraft is at a negative potential. The energy gained increases the ram component of the ion velocity distribution, which in turn bends the ion beam toward the aperture normal. This is analogous to what happens to the beam when passing from the grounded grid in front of the suppressor to the suppressor. The difference being, in the case of sheath acceleration, that the angle of the beam is not corrected by forcing the ions to climb an equivalent energy hill as in the case of the suppressor. To correct for this acceleration, the velocity in Eq. (6) can be replaced by a corrected velocity,

$$
v_{\mathrm{o}}^{\prime}=\sqrt{v_{0}^{2}+\frac{2 q V_{s}}{m}},
$$

where the prime again denotes the corrected quantity and $V_{s}$ is the spacecraft potential. An alternative approach is to mitigate the effect 
of spacecraft charging by measuring the floating potential of the plasma and using this measurement to either correct for the effect in data processing or electronically float the ground potential of the instrument. ${ }^{10}$

\section{DESIGN REQUIREMENTS}

The focus of the GRIDS instrument on the petitSat mission is to characterize plasma enhancements as well as the background ionosphere. This goal flows down to the measurement requirements for the instrument, which defines the overall design requirements. The measured quantities required to complete the science goals of the petitSat mission are the plasma density fluctuations, changes in the light $(\mathrm{H}+, \mathrm{He}+)$ to heavy $(\mathrm{O}+)$ ion concentration, and the three-dimensional plasma drift vector. The GRIDS is responsible for measuring all three of these quantities.

The plasma density fluctuations are measured during the IDM mode as well as at the beginning of the RPA mode. The total flux of ions to the collectors of GRIDS, combined with velocity information obtained during RPA mode, enables the determination of the overall ion density. The total current collected is related to the ion density, as shown in Eq. (1). Based on the nature of plasma enhancement characteristics, plasma density measurements over a range of $10^{3}-10^{7} \mathrm{~cm}^{-3}$ are required. In addition, these changes need to be characterized with an accuracy and precision of $10 \%$. The ratio of light to heavy ions is determined using RPA mode. As the voltage is increased on the retarding grids, the lower energy ions are screened out. Since all ions are moving with equal bulk velocities, lighter ions have lower energy than heavier ions. These conditions lead to two distinct plateaus in the I-V curve when light and heavy ions are present. If the signal to noise ratio is sufficiently high, the current collected at each of these plateaus allows for the calculation of the light/heavy ion density via the same formula used for overall ion density. The science of the petitSat mission requires light/heavy ion species densities to be characterized over a range of $10^{3}-10^{7} \mathrm{~cm}^{-3}$ with an accuracy and precision of $10 \%$.

The bulk plasma drift vector is the most challenging measurement made by the GRIDS sensor. It requires the combined analysis
TABLE I. Measurement requirement for GRIDS.

\begin{tabular}{lccc}
\hline \hline Parameter & Range & Accuracy & Precision \\
\hline Density & $10^{3}-10^{7} \mathrm{~cm}^{-3}$ & $10 \%$ & $10 \%$ \\
Composition & $10^{3}-10^{7} \mathrm{~cm}^{-3}$ & $\begin{array}{c}10 \% \\
10 \%\end{array}$ \\
Velocity & $500-500 \mathrm{~m} / \mathrm{s}$ & $10 \mathrm{~m} / \mathrm{s}$ relative & $10 \mathrm{~m} / \mathrm{s}$ \\
\hline \hline
\end{tabular}

of data from both the IDM and RPA modes. As described earlier, the RPA mode allows for the determination of the component of the drift vector normal to the front face of the instrument. The other two orthogonal drift vectors are determined in the IDM mode, as described in Sec. IV. Science closure on the mission requires that these drifts be determined over a range of $-500-500 \mathrm{~m} / \mathrm{s}$ with a $10 \mathrm{~m} / \mathrm{s}$ precision. A summary of these measurement requirements is presented in Table I.

These measurement requirements flow down to several interrelated instrument requirements. Some of these can be described easily because the flow-down is straightforward. Others are more difficult to describe with a precise flow-down because the fundamental quantities obtained from an individual RPA I-V curve are inferred using a nonlinear curve-fitting routine. This means that there are complex and intertwined relationships between various instrument design features and the resulting quantities produced. ${ }^{12}$ Furthermore, these relationships are dependent on the particular curve-fitting routine used and on the initial estimates used to seed the curve-fitting routine. For this reason, some requirements are derived from a "best-practices" perspective that has been gained from studying previous successful missions that utilized an RPA. A summary of the design requirements can be found in Table II.

Additionally, the petitSat mission will utilize a $6 \mathrm{U}$ cubeSat bus, so GRIDS must be compatible with this form factor. This requires the GRIDS to be less than $1 \mathrm{U}$ in volume, with an overall mass less than $1 \mathrm{~kg}$ and a total power consumption less than $1 \mathrm{~W}$. The bus should be able to maintain internal temperatures in the $-5{ }^{\circ} \mathrm{C}$ to $50^{\circ} \mathrm{C}$ range, so GRIDS must be functional within that range.

TABLE II. Design requirements for GRIDS

\begin{tabular}{|c|c|c|}
\hline Design parameter & Requirement & Rationale \\
\hline Measured current range & $400 \mathrm{pA}-4 \mathrm{uA}$ & $\begin{array}{l}\text { These current ranges correspond to the per-collector segment current expected } \\
\text { over the range of densities given in Table I. }\end{array}$ \\
\hline Measured current accuracy & Greater of $50 \mathrm{pA}$ or $2.5 \%$ & $\begin{array}{l}\text { This level of accuracy allows for velocities determined via curve-fitting to } \\
\text { produce the accuracy and precision required in Table I. }\end{array}$ \\
\hline Samples per I-V curve & 16 or more & $\begin{array}{l}16 \text { samples per curve are near the low-end of previously flown RPAs. More } \\
\text { samples per curve allow for more robust curve-fitting. }\end{array}$ \\
\hline Retarding voltage range & $0-10 \mathrm{~V}$ or more & $\begin{array}{l}10 \mathrm{~V} \text { is enough to screen out a majority of } \mathrm{O}^{+} \text {ions for typical orbital velocities in } \\
\text { the region of interest }\end{array}$ \\
\hline Suppressor bias & $-5 \mathrm{~V}$ or less & $\begin{array}{l}-5 \mathrm{~V} \text { is sufficient to reject nearly all ambient electrons while also returning } \\
\text { photoelectrons to the collector surfaces }\end{array}$ \\
\hline Acceptance angle & $4.1^{\circ}$ or more & $\begin{array}{l}\text { Acceptance angles larger than } 4.1^{\circ} \text { ensure that ions moving within the velocity } \\
\text { ranges specified in Table I are collected. }\end{array}$ \\
\hline Measurement cadence & $2 \mathrm{~s}$ or less & Provides the $\sim 15 \mathrm{~km}$ spatial resolution required by the mission. \\
\hline
\end{tabular}




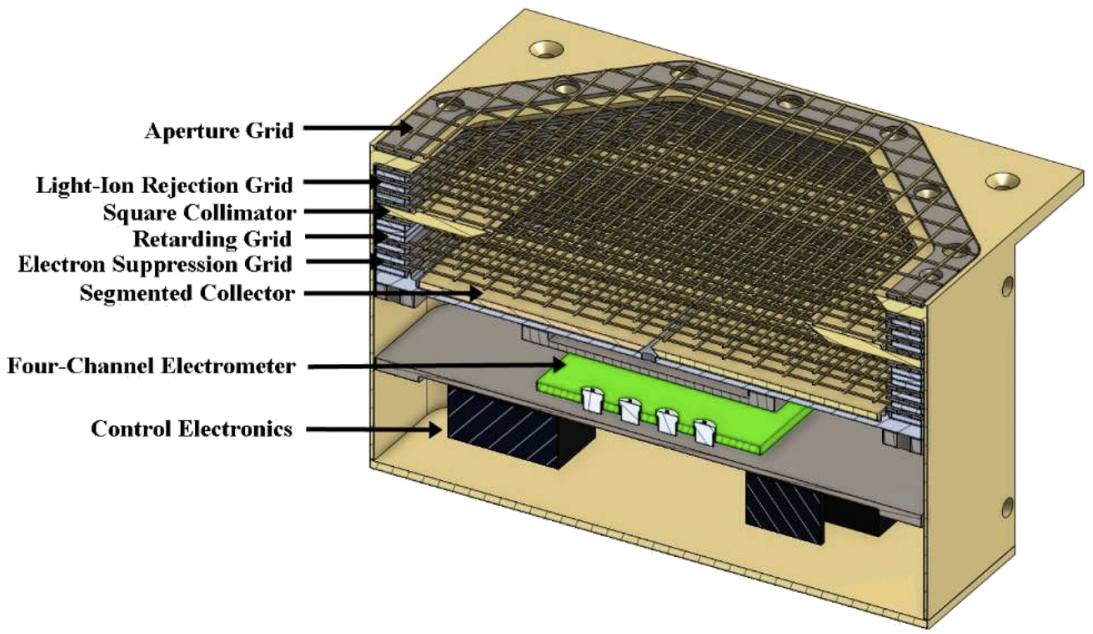

FIG. 3. A cross-sectional view of GRIDS.

\section{MECHANICAL DESIGN}

GRIDS is a self-contained sensor with only one bus-side connector on the backside of the instrument. The grid stack and aperture are only exposed on the front (ram) side. A cross-sectional view of the instrument computer aided design (CAD) model is presented in Fig. 3. The grid stack from top to bottom consists of the aperture grid flush with the front of the instrument, the light ion rejection grid, the square collimator (sandwiched between two grids), a retarding grid, and finally the segmented collector. Behind the collector assembly is the smaller of the two electrical boards that house the four-channel electrometer, followed by the main electrical board that provides all the other necessary functionalities. The two electrical boards are connected to the collector segments and biased grids through small cables attached to screws that are connected to the appropriate surface. A side view of the instrument with the outer sleeve removed is shown in Fig. 4.

The octagonal design allows for repeatable and consistent relative alignment of the grids with each other and with the square

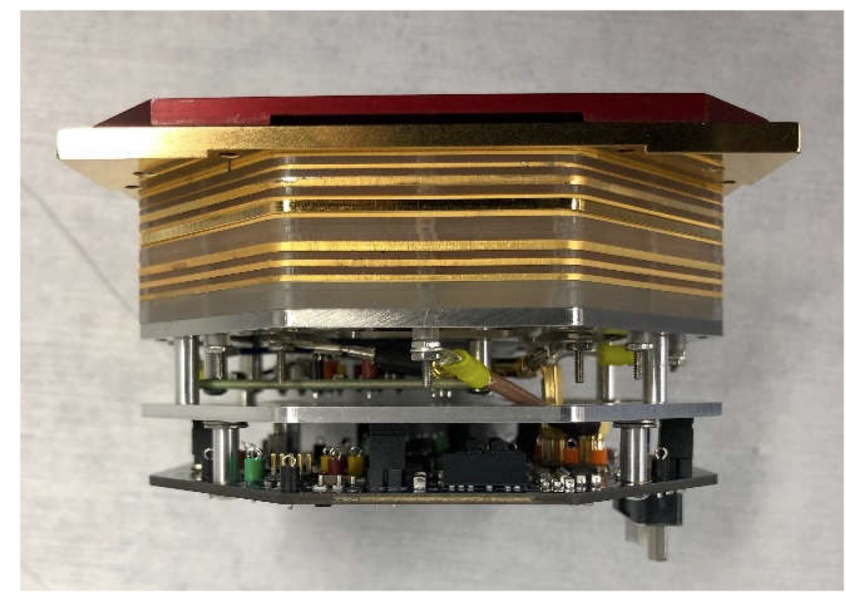

FIG. 4. The interior of the GRIDS sensor. aperture. Additionally, the stacked nature of the instrument components allows for ease of assembly/disassembly and allows the fragile grids to be isolated from the other components to reduce the risk of damage during testing or integration.

Overall, the sensor is $95 \times 95 \times 54 \mathrm{~mm}^{3}\left(487 \mathrm{~cm}^{3}\right)$ excluding the slight protrusion of the connector on the back of the instrument. The total mass of the sensor is $509 \mathrm{~g}$, excluding the cable harness that connects the instrument to the bus. GRIDS will be mounted on the petitSat bus using the mounting holes on the sides of the instrument (four of which can be seen in Fig. 1) such that the front of the sensor is flush with the ram face of the spacecraft (the $3 \mathrm{U}$ face for petitSat).

\section{ELECTRICAL DESIGN}

The GRIDS sensor utilizes two electrical boards. The smaller of the two houses the four-channel electrometer and can be seen in Fig. 5. The larger mainboard contains all the other necessary circuitry to perform all other instrument functions. The GRIDS mainboard is shown in Fig. 6. These two boards are connected through a 21-pin nano-D connector that is mounted on each board.

The electronics on the two boards carry out five major functions:

1. Measure the four currents collected by the collector segments.

2. Generate the voltages needed by the ion-rejecting grid, the retarding grid, and the suppressor grid.

3. Generate all power rails needed for the internal circuitry of the instrument from the bus input voltage.

4. Monitor various status and health quantities for housekeeping purposes.

5. Digitize and transmit the collected data to the bus.

The first of these functions is carried out exclusively on the daughterboard. The electrometer on this board uses a chargeintegration method to measure the small currents collected by the four collector elements. In each channel of the electrometer, a small capacitor is charged up to a known voltage. That capacitor is then connected to the collector segment and allowed to discharge through 


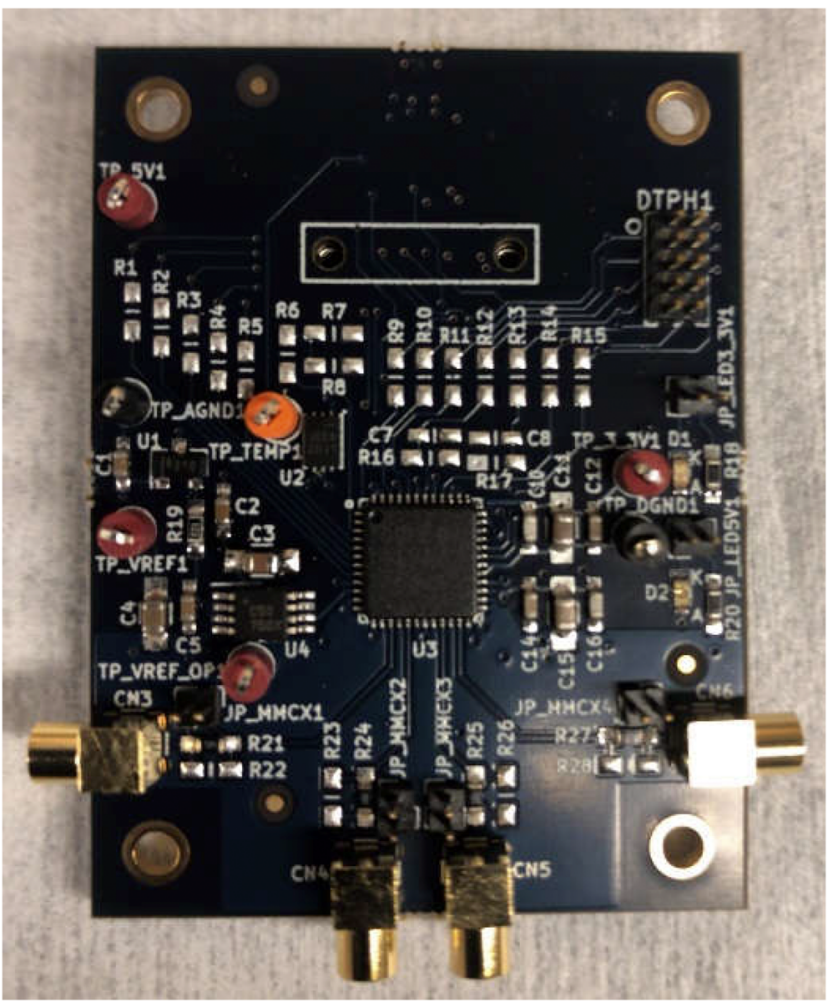

FIG. 5. The GRIDS daughterboard.

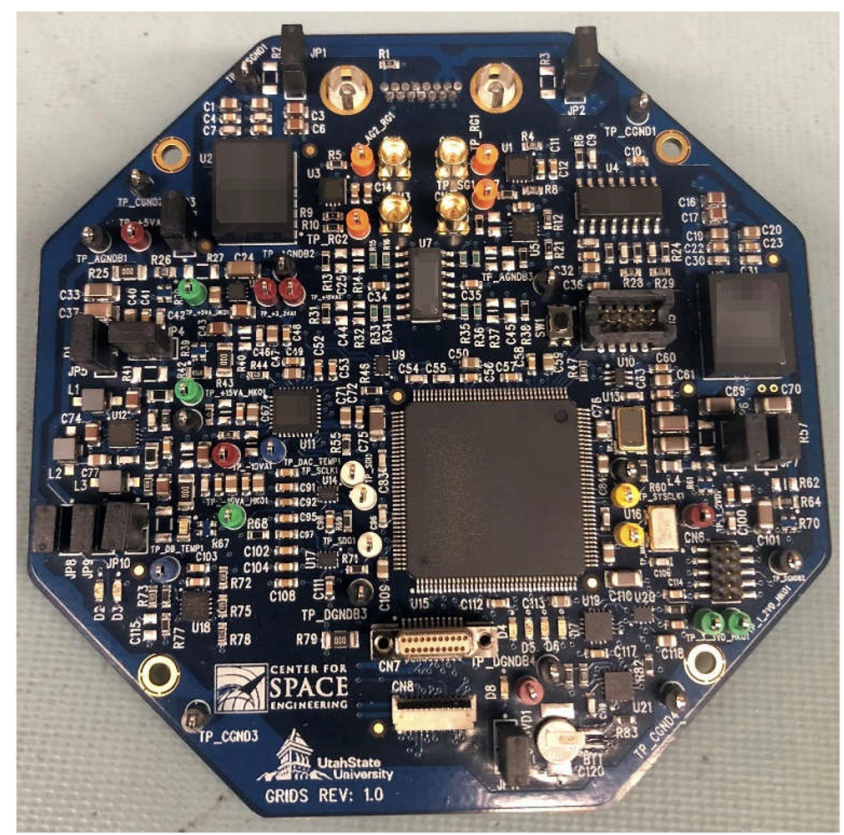

FIG. 6. The GRIDS mainboard. the collected current for some known amount of time. It is then disconnected from the collector and the remaining capacitor voltage is measured. This allows the average current collected over the integration time to be calculated. Autonomously varying the integration time based on the measured current magnitude allows vastly different currents to be measured without compromising noise characteristics. This method is a good match for this application because it allows a large input current range to be measured while maintaining low-end noise to a level below 100 pA. Additionally, the charge integration method is not sensitive to changes in the input current on scales less than the integration time, but the bulk flow of the plasma is not expected to change significantly on these timescales.

The remaining four key functions are carried out by using the mainboard circuitry. Power is brought in through the main 15pin connector on the back of this board. It is fed into two isolated DC/DC converters that in concert with a few linear regulators provide all of the internal voltages required for the instrument. This design allows the GRIDS electronics to be isolated from other bus systems while providing flexibility on the input voltage required. Nominally, $12 \mathrm{~V}$ is fed to the instrument but it can handle unregulated voltages between $3.3 \mathrm{~V}$ and $32 \mathrm{~V}$ while still operating nominally. Slight differences in power consumption arise for different bus voltages because the DC/DC converter efficiency is input-voltage dependent. The analog and digital supply voltages are separated by using two isolated DC/DC converters to help reduce the noise present near the sensitive analog components. Internally, seven voltages are produced to run the various electrical components. For the digital components, $3.3 \mathrm{~V}$ and $1.2 \mathrm{~V}$ are required. The analog components require $3.3 \mathrm{~V}, 5 \mathrm{~V}, \pm 15 \mathrm{~V}$, and a reference voltage of $4.096 \mathrm{~V}$ for the analog to digital converter. In addition to being isolated from the bus power system, the analog and digital voltages are isolated from one another by using separate DC/DC converters as well as separate ground and power planes within the printed circuit board (PCB). A block diagram of the power generation circuitry can be seen in Fig. 7.

The grid drive voltages are generated using a four-channel digital-to-analog converter (DAC) feeding four analog filters to help stabilize the voltages that are passed to the grids. The four voltages allow for the use of two independently driven retarding grids, which adds redundancy as well as the flexibility to switch between single and double retarding grid configurations.

Housekeeping data are collected through a series of analog to digital converters (ADCs). These use various sensor ICs to measure each of the power rail voltages, the current being used on each power rail, and the temperature at various points on each board. In addition, two other quantities are included in the housekeeping. A real-time-clock chip is polled at regular intervals to help ease data processing and ensure that measurements are analyzed in the correct time sequence. A three-axis magnetometer is also included to identify data taken during attitude maneuvers, since these may be compromised.

All of these functions are controlled by a field-programmable gate array (FPGA) on the mainboard. In addition to controlling the other components on the boards, the FPGA is also responsible for ordering the data collected into packets and transmitting them to the bus. One data packet is sent to the bus every $2 \mathrm{~s}$ via an RS422 interface. This communication bus is electrically isolated from the power bus. 


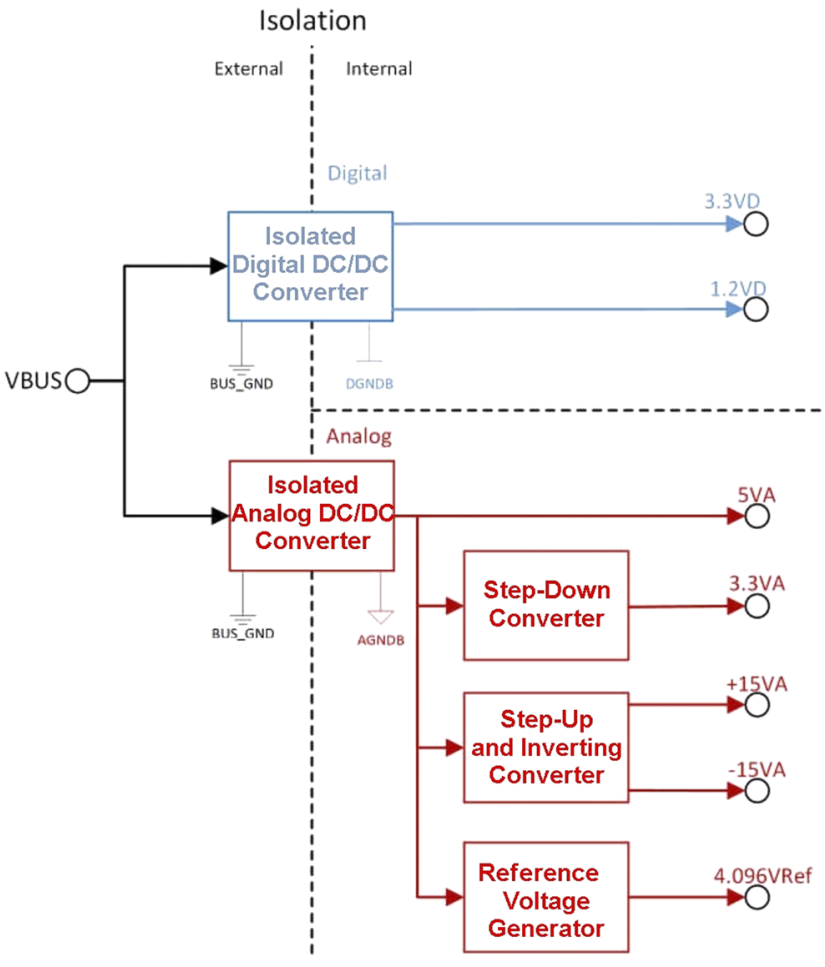

FIG. 7. Block diagram of the GRIDS power circuitry.

\section{DATA STRUCTURE}

The GRIDS instrument produces a packet every two seconds. Each packet consists of a header and measurement data. The header is made up of status, timing, and housekeeping information and is a fixed 66 bytes ( 8 bits/byte). The measurement data contain a voltage measurement for each of the four driven grids plus four current measurements (one from each collector) for each of the 40 RPA points and 10 IDM points (50 total). The voltage measurements are two bytes each, while each current measurement is 20 bits. Additionally, an integration timer value is included to denote which current-measurement range the instrument was in for a particular measurement, resulting in 1000 bytes of measurement data per packet. Altogether, GRIDS produces one 1066 byte packet every other second for a total data rate of $4262 \mathrm{bit} / \mathrm{s}$. A depiction of the packet structure is shown in Fig. 8.

\section{LABORATORY VALIDATION PROCEDURE}

The GRIDS sensor has been validated in two different sets of laboratory tests. The first set of tests were done on the GRIDS electronics in a benchtop setting. These tests were designed to verify that the grid drive circuitry produces the correct voltages and the housekeeping data were accurate and to characterize the current sensing capability of the four-channel electrometer. The second set of tests were carried out in a plasma-beam vacuum chamber to validate the end-to-end functionality of the instrument.

To verify that the grid-drive circuitry functions correctly, a four-channel oscilloscope was attached to output of the four grid drive channels and voltage data were captured over several measurement cycles. These voltages are also captured internally by the GRIDS electronics and included in the generated data packets. These measurements are used to verify voltage levels and determine if the correct voltages are being generated at the correct times. Finally, these oscilloscope measurements allow for the characterization of the rise time produced by the grid drive circuitry before the voltage on the grids stabilizes and to show that it is consistent with the $1 \mathrm{~ms}$ design goal. The measured grid voltages are shown in Fig. 9.

\begin{tabular}{|c|c|c|c|c|c|c|c|c|c|c|c|}
\hline \multicolumn{10}{|c|}{ GRIDS Data Packet (1066 Bytes) } \\
\hline 14 Bytes & 52 Bytes & 8 Bytes & 10 Bytes & 2 Bytes & 8 Bytes & 10 Bytes & 2 Bytes & 940 Bytes & 8 Bytes & 10 Bytes & 2 Bytes \\
\hline Packet Status & Housekeeping & $\begin{array}{c}\text { Grid } \\
\text { Voltages }\end{array}$ & $\begin{array}{c}\text { Collected } \\
\text { Current Data }\end{array}$ & $\begin{array}{c}\text { Integration } \\
\text { Timer }\end{array}$ & $\begin{array}{c}\text { Grid } \\
\text { Voltages }\end{array}$ & $\begin{array}{c}\text { Collected } \\
\text { Current Data }\end{array}$ & $\begin{array}{l}\text { Integration } \\
\text { Timer }\end{array}$ & $\ldots \ldots$ & $\begin{array}{c}\text { Grid } \\
\text { Voltages }\end{array}$ & $\begin{array}{c}\text { Collected } \\
\text { Current Data }\end{array}$ & $\begin{array}{l}\text { Integration } \\
\text { Timer }\end{array}$ \\
\hline
\end{tabular}

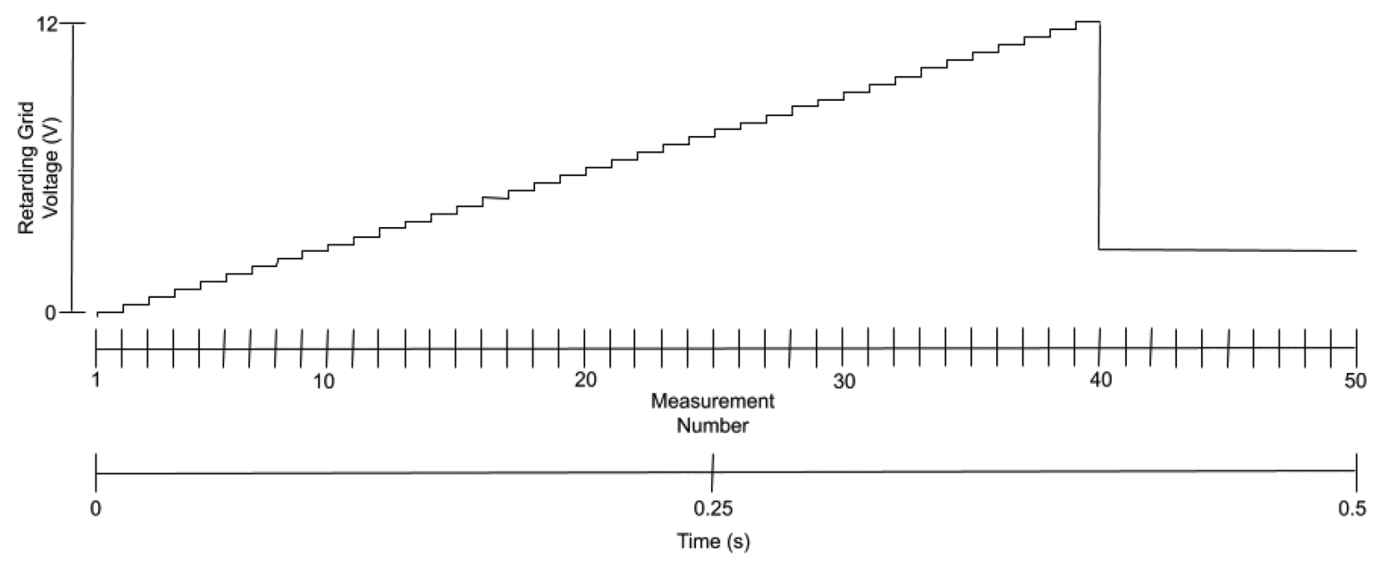

FIG. 8. The packet structure for the GRIDS instrument 


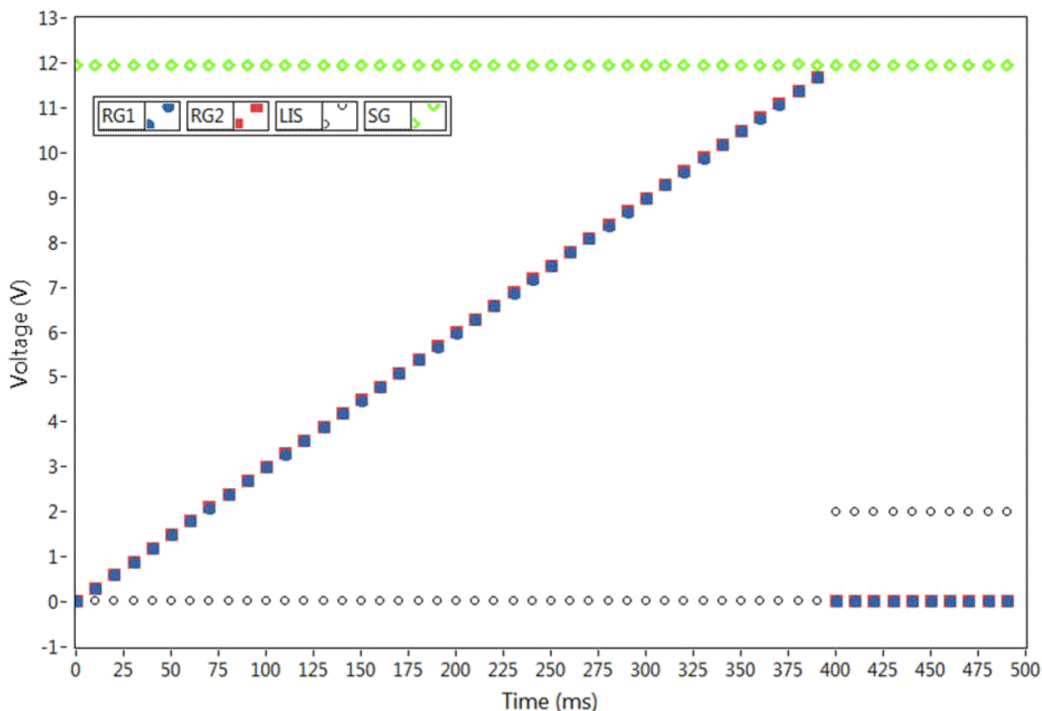

FIG. 9. Grid voltage measurements of the four driven grids in the instrument. The light ion screen (LIS) remains low during RPA mode and is set at $2 \mathrm{~V}$ during IDM mode. The retarding grids (RG1 and RG2) are swept from $0 \mathrm{~V}$ to $12 \mathrm{~V}$ during the RPA mode and held at $0 \mathrm{~V}$ during the IDM mode. The suppressor grid (SG) is held at a constant $-12 \mathrm{~V}$. (Note: the suppressor voltage is inverted in the plot to compress the scale).
The housekeeping data are comprised of the internal voltage levels, current draw on each bus, and various internal temperatures. The voltages generated by the power supply circuitry are compared against external measurements made using a high-quality multimeter. The currents drawn on each individual voltage rail are verified by inserting a current meter in each current path. Temperature sensors placed throughout the electronics' boards are verified using an external optical temperature sensor.

A high precision low-level current source is used to characterize the four-channel electrometer. For each channel, currents over the full range of possible input levels are driven into each channel. The input and measurement current values can be compared to characterize the linearity of the electrometer as well as its precision and noise levels. A plot of measured current vs input current is shown in Fig. 10. With no current input, the noise floor of the inputs is measured to be $52 \mathrm{pA}$.

In addition to the bench-testing and validation procedures, the performance of the GRIDS instrument has been validated through additional tests carried out in a vacuum chamber designed to simulate the near-Earth geospace environment. These tests utilize a newly developed ion source designed specifically for the validation of thermal ion instruments. ${ }^{13}$ The ion source uses energetic electrons emitted by heated tungsten filaments to ionize a portion of the ambient neutral gas molecules that enter through an opening at the back of the source. Electrically biased surfaces within the source ensure that the energetic electrons interact with the neutral gas and that the ions created are pushed out through the aperture. By controlling the internal biases, the mean energy and the directed velocity of the ions are controlled so that the result is a focused beam of ions that enter the GRIDS aperture normal to the front face of the instrument. The vacuum pressure maintained in the system during each test creates total ion flux rates to the GRIDS sensor that closely approximate the fluxes and incident velocities encountered in the circular low-Earth orbit (LEO). Figure 11 shows a photograph of GRIDS and the ion source in the IDM-validation test-configuration.
Laboratory validation tests are carried out separately for the RPA and IDM modes of the GRIDS instrument because the ion source is configured differently for the two types of tests. For example, the angle of the aperture relative to the incident ion beam must be varied to validate the IDM mode of operation, but this is not required for validation of the RPA-mode. The incidence angle variation for the IDM-mode tests is implemented using a rotary motion platform that is remotely controlled via a LabView ${ }^{\mathrm{TM}}$ interface. In both types of validation testing, the ion currents to the GRIDS instrument are in the nano-amp range, and the ions enter the aperture of GRIDS at speeds ranging from $6 \mathrm{~km} / \mathrm{s}$ to $9 \mathrm{~km} / \mathrm{s} .{ }^{13}$ The ion speeds are varied by adjusting the voltages applied to various conducting surfaces inside the source. The validation efforts using the

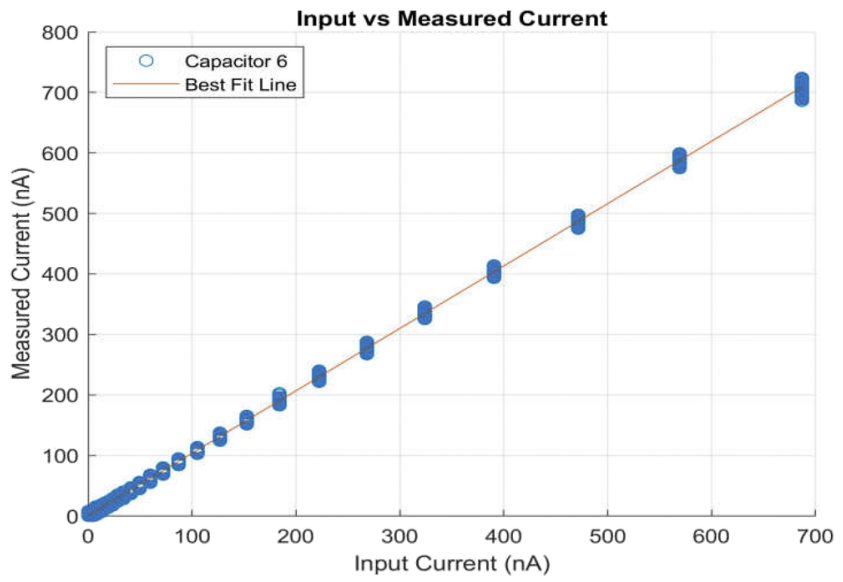

FIG. 10. Input current vs measured current for one of the GRIDS current channels showing good linearity of the measurement over a wide range of input values. 


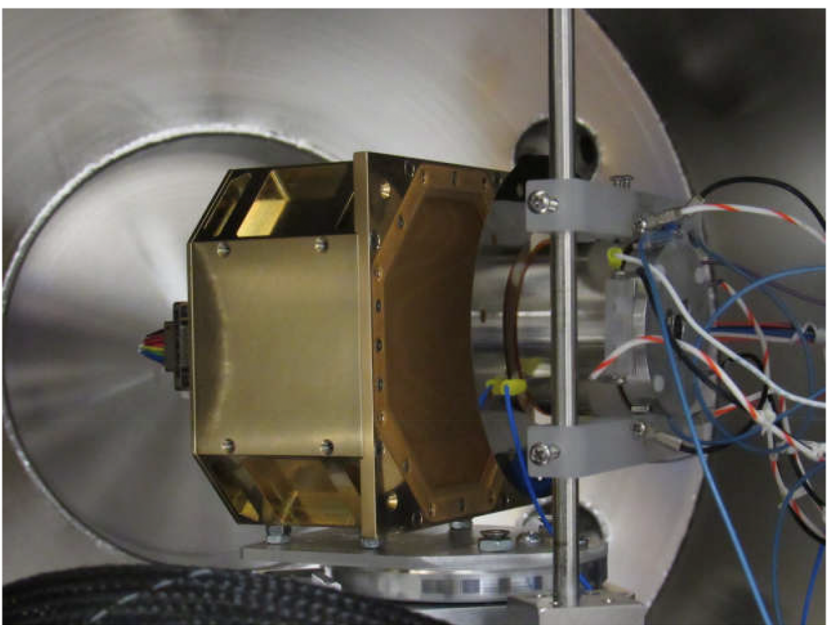

FIG. 11. The GRIDS instrument (left) is shown mounted on a remotely controlled rotary table adjacent to the ion source (right) that produces fluxes comparable to those encountered in low-Earth orbit.

ion source can successfully mimic some of the conditions encountered in the flight environment, specifically the ion velocity and total flux into the GRIDS aperture. Other conditions, such as the energy distribution, $\mathrm{O}+$ composition, and photoemission due to sunlight cannot easily be created in a laboratory vacuum system. Since these validation efforts are performed under conditions that mimic LEO conditions, they raise the NASA technology readiness level (TRL) of the GRIDS instrument to 6 .

\section{LABORATORY VALIDATION RESULTS}

To validate the capabilities of GRIDS in a flight-like environment, the instrument is installed in the vacuum chamber with its aperture facing the ion source, as shown in Fig. 11. The vacuum level and voltage biases internal to the source are then adjusted so that the total flux of ions into the aperture of GRIDS is comparable to the flux levels expected during LEO flight in the ionosphere. The data packets generated by GRIDS are read using a LabView program that displays key state-of-health data and science measurements to a monitor while simultaneously archiving a file containing all the transmitted packets. No averaging or smoothing is performed on these data so that the integrity of the GRIDS data is not compromised or misinterpreted.

\section{A. RPA-mode test results}

Validation of the RPA-mode is accomplished in two steps. First, the vacuum pressure in the chamber is adjusted to vary the flux into the aperture of GRIDS. The ion source creates ions from the background gas in the chamber, so increasing the pressure of the neutral gas in the chamber increases the flux of ions into the GRIDS aperture. The first current sample (at a retarding voltage of zero) in each current-voltage (I-V) characteristic is confirmed to vary linearly with the flux levels emitted by the ion source, thereby demonstrating that variations in the ion density in the orbit can be correctly

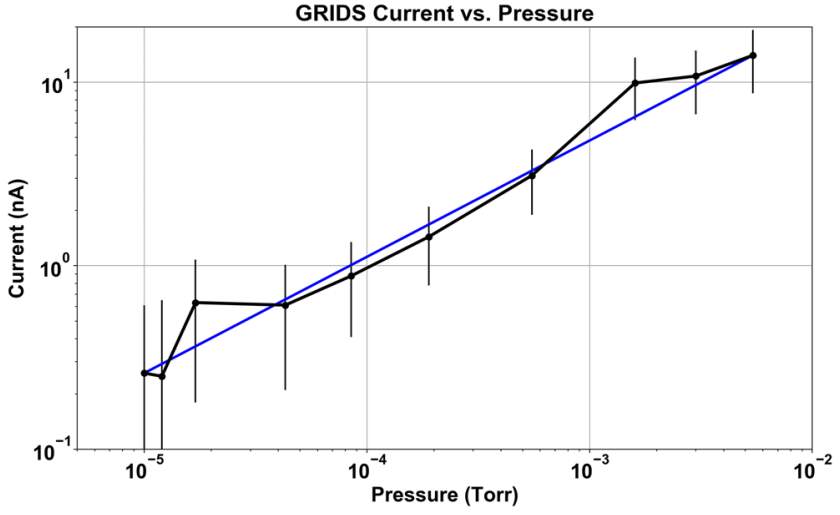

FIG. 12. Linear variation of GRIDS currents over a range of background pressures.

estimated from the I-V curves. Figure 12 shows an example from such a test in which the first five current samples from each retarding grid voltage sweep (currents at the five lowest retarding voltages) are averaged together to reduce the effects of electronic noise. The three pressures plotted are $8.3 \mu$ Torr, $1.0 \mathrm{mTorr}$, and $4.5 \mathrm{mTorr}$, which convert to meter, kilogram, and second units of $1.1 \mathrm{mPa}, 133 \mathrm{mPa}$, and $598 \mathrm{mPa}$.

Next, the internal biases in the ion source are varied to alter the speed at which the ions enter the aperture of GRIDS. As the speed increases, the I-V curves obtained in RPA-mode should shift to the right because higher voltages on the retarding grids within the instrument are required to attenuate the ion flows to the collector elements within GRIDS. This behavior is shown in Fig. 13, where the I-V curves have been normalized to allow self-consistent comparisons between the distinct data curves. The four curves shown in the figure correspond to four different internal voltage settings within the ion source. These voltages control the speed of the emitted beams, where larger voltage settings correspond to faster ion beams. Multiple trials at each beam speed allow meaningful averages and

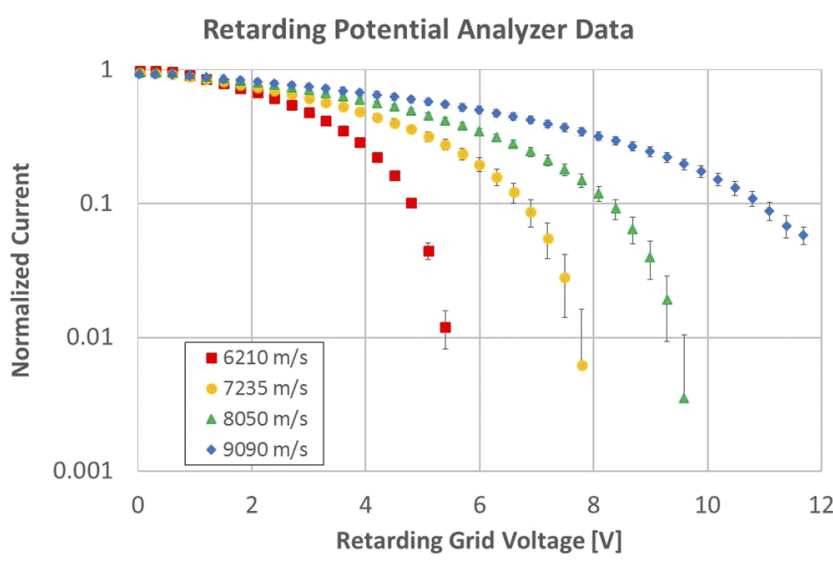

FIG. 13. Four I-V curves corresponding to increasing ion beam speeds into the aperture of GRIDS. 
standard deviations to be calculated, as shown in the figure. The speeds shown in the figure are derived from the I-V curves using the procedure described by Robertson et al. Essentially the point in each I-V curve where the current is one half its maximum value is used to estimate a ram speed at which the ions enter the GRIDS aperture. This is valid because the half-current point corresponds to the retarding voltage at which half the ion distribution is rejected by the voltage on the retarding voltage grid. The retarding voltage of this half-current point in each $\mathrm{I}-\mathrm{V}$ curve can be called $\mathrm{V}_{0}$. The mass of the ions is known because we control the composition of the neutral gas in the chamber, so we determine the speed by simply equating the kinetic energy of the collected ions to the potential energy $\mathrm{qV}_{0}$, where $\mathrm{q}$ is the charge on the ion. The speeds listed in the plot index are obtained by solving for the velocity.

It is worth noting in Fig. 13 that the I-V curves are not representative of Maxwellian energy distributions because the ion beams emitted by the source are not in thermodynamic equilibrium. The curves shown in Fig. 13 are typical of I-V curves measured for beams produced in laboratory vacuum chambers. The mean-free paths of the ions at the pressures required to achieve LEO-like fluxes are large relative to the size of the source and its distance from the aperture of GRIDS, and the potential gradients internal to the source lead to the production of ions over a range that covers many volts. In the ionosphere, the situation is different because the ions have time to collide with each other and with other particles as they relax to a distribution with a well-defined temperature. The result is that both the flow speed and flux of ions into GRIDS are consistent with LEOlike ion distributions, but the distribution itself is broader in energy than it is in the ionosphere. Thus the response of GRIDS to the speed and density of the ion beam can be validated, but the ion temperatures inferred from I-V curves like those shown in Fig. 10 are not meaningful.

Despite the non-Maxwellian energy distributions, the beam speeds can be estimated from I-V curves like those shown in Fig. 13. This is done by recognizing that the retarding voltages at which the current measurements in each I-V curve reach half their maximum value correspond to half of the incident ions being rejected. Under the assumption that the ion distribution function is roughly symmetric, the voltage at this half-current point corresponds to the average velocity of the ions comprising the beam. ${ }^{14}$ Equating the potential energy of the ions to the voltage at this half current point therefore gives a reasonable estimate of the speed of the ion beam as it enters the aperture of GRIDS. Figure 14 shows the resulting speeds along with the voltages (right axis) of the half-current points. Satellites in stable, circular orbits have speeds near $8 \mathrm{~km} / \mathrm{s}$ in the Earth-fixed frame, and the figure shows that the velocities of the ion beams used in testing GRIDS have comparable magnitudes.

\section{B. IDM-mode test results}

The data shown above verify that the RPA-mode measurements made by GRIDS accurately reflect the state of the ion beam in laboratory vacuum-chamber tests. The final validation test for GRIDS involves validating its sensitivity to the arrival angle of the incident ion beam. For this test, it is important that the beam emitted from the source be collimated, ideally with an outward velocity normal to the plane of the aperture. Once this condition is realized, the rotary table on which the GRIDS is mounted can be adjusted over a range

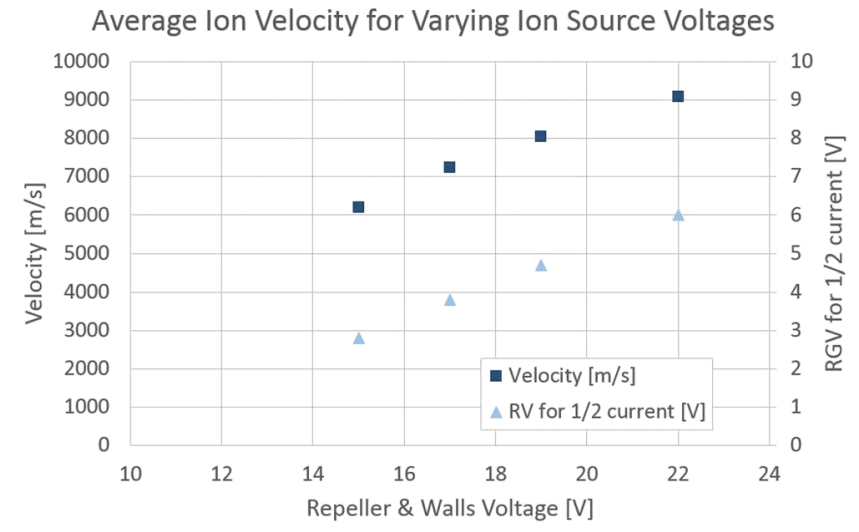

FIG. 14. Retarding grid voltages at the half-maximum current values (right axis) and ion beam speeds (left axis) derived from them for the four $\mathrm{I} V \mathrm{~V}$ curves shown in Fig. 10. The beam speeds are comparable to those observed by a satellite in LEO

of angles corresponding to realistic LEO-like velocities orthogonal to the GRIDS aperture normal. The velocity vector of the ion beam is thereby rotated relative to the GRIDS aperture, producing a measurable cross-track velocity. This two-dimensional velocity vector is analogous to ion drifts in the plane orthogonal to the velocity vector of a LEO satellite, where it is understood that the instrument is mounted on the leading surface (ram-face) of the spacecraft, and it is assumed that the thermal and directed drift velocities of the ions are much less than the relative velocity between the ambient ions and the spacecraft.

An ideal test condition is one in which the ion flux produced by the source is perfectly uniform across the aperture of GRIDS and where the alignment between the beam velocity vector and the normal to the instrument aperture is exactly parallel. Neither of these conditions can be achieved in practice. For example, since two different hand-wound filaments are used in the ion source to create the beam, neither the filaments themselves nor the flux of ionizing electrons they produce will be identical. The result is a non-uniform flux of ions distributed across the aperture. The non-uniformity can be observed by passing a small current-collecting probe diametrically across the face of the aperture; the results of two such measurements at different distances from the aperture are shown in Fig. 15. The left panel of the figure shows a gray-scale image of the beam flux interpolated from probe measurements at several different distances from the aperture. The aperture of the source is at the left, and the particles are emitted toward the right in this figure. The right panel shows current data from a particular set of current-probe measurements at distances of $0.635 \mathrm{~cm}$ and $3.81 \mathrm{~cm}$ from the aperture grid.

The non-uniformity of the emitted ion beam coupled with small alignment errors between the ion source and the GRIDS instrument will produce an offset between the angle inferred from the GRIDS collector data and the angle setting of the rotary table on which the GRIDS is mounted. In other words, the currents $\mathrm{I}_{1}$ and $I_{2}$ in Eq. (2) will not be the same even when the beam velocity is perfectly normal to the aperture plane because the beam that is produced by the ion source is not perfectly symmetric around the centerline of the aperture (see Fig. 15). Equation (3) shows how small 

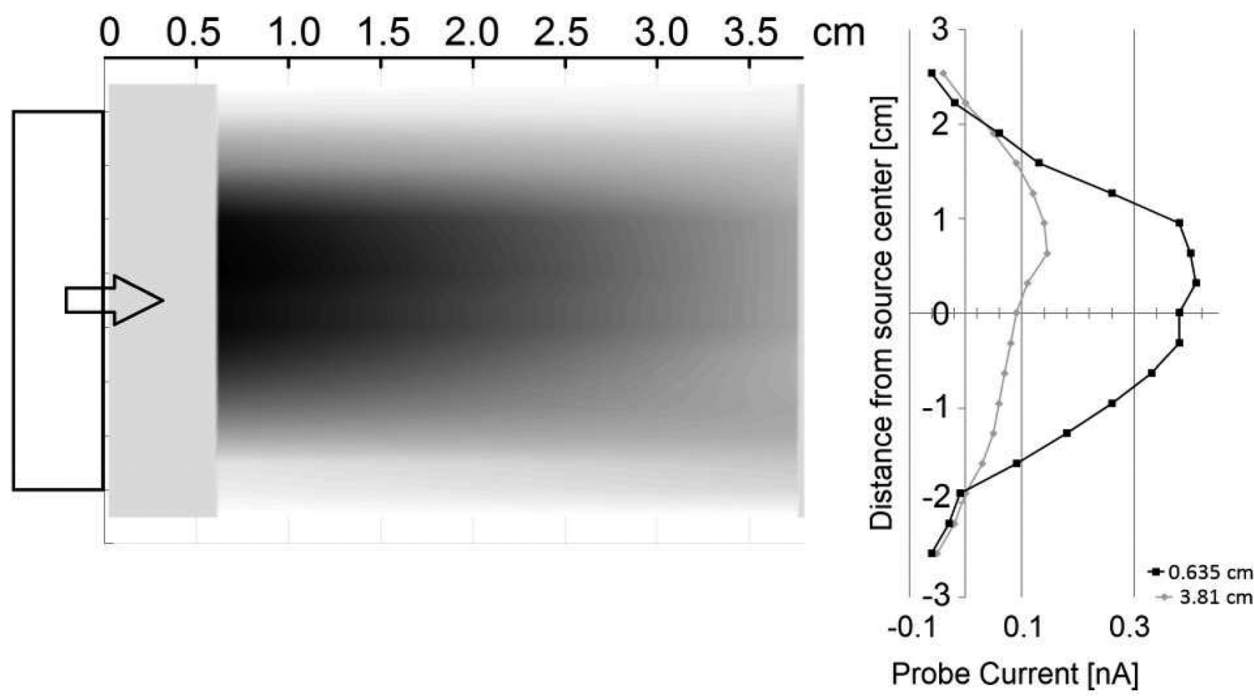

variations in the ratio of the two currents are related to the measured incidence angle. Despite these shortcomings, the functionality of the GRIDS IDM measurement can be assessed through vacuum chamber testing with the rotary table.

The objective of the angular sensitivity testing is simply to show that the ratio of currents to the collectors inside GRIDS varies as the angle between the ion beam and the normal to the aperture of the instrument is changed. The ion source is not being used to calibrate the GRIDS device, but to validate that it is sensitive to changes in the flow velocity of the ions along three separate axes. Calibration is done on the bench using a precision current source and known resistive dividers corresponding to specific current ratios driven to each collector. Figure 16 is therefore not a calibration but simply an end-to-end validation of the instrument in a flowing ion beam.

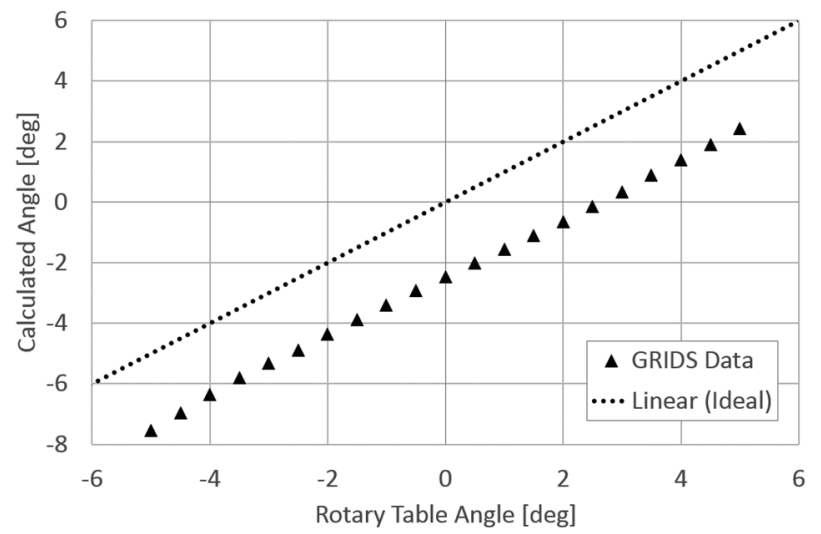

FIG. 16. The horizontal axis shows the angle of the rotary table relative to the ion beam velocity, and the vertical axis shows the angle calculated from the measured current ratios. The offset is related to the non-uniform ion beam and possible misalignment between the apertures of the GRIDS and the ion source. The smaller dotted line shows the expected result for a perfectly uniform ion beam with no alignment error between the devices.
The figure shows this by measuring the incidence angle of the beam using Eqs. (2) and (3). The horizontal axis in the figure is the nominal angle of the ion beam relative to the aperture of GRIDS, which is set by commanding the rotary table to a specific angle. The vertical axis shows the angle calculated from the ratios of the measured currents. The straight dotted line that passes through zero is the ideal case that would be achieved for a uniform beam and perfect alignment between GRIDS and the ion source. Comparison of the measured angles with the idealized case clearly shows that GRIDS is responsive to changes in the incidence angle of the ion beam, despite the imperfections in the source stimulation discussed above.

Data of the type shown in the figure are collected twice, once to characterize the response to the elevation angle of the beam and once for the azimuthal variation. Thus, we verify that the GRIDS instrument is sensitive to the angle of arrival (cross-track velocity) in two orthogonal planes, both of which contain the vector normal to the instrument aperture. In practice, this is accomplished by measuring the response to incidence angle changes in one plane and then opening the chamber and rotating the GRIDS instrument by $90^{\circ}$ relative to the ion source before repeating the test. In this way, the full angular sensitivity of the GRIDS instrument is verified.

In flight, the ion flux across the diameter of the instrument aperture will be much more uniform than it is in the laboratory tests because the high viscosity of the ionospheric plasma cannot support short-scale velocity gradients. As a result, the scale lengths over which the ion density and cross-track flow vary in the ionosphere are much larger than the aperture of GRIDS, so arrival angle measurements in the orbit will yield data that can be interpreted accurately using the bench-calibration curves for each distinct collector channel in conjunction with Eqs. (2) and (3).

The thermal speeds of the ions encountered in orbit are much smaller than the orbital speed of the satellite, so the arrival angle measurements made in IDM mode are very close to the actual arrival angle of the gas relative to the velocity vector of the satellite. The known satellite velocity, coupled with the ram ion drift measurements inferred from the RPA-mode data, allows the actual arrival 
angle to be determined very accurately as long as the satellite attitude is well determined and the sheath potential is planar across the aperture of GRIDS. Together, the IDM and RPA-modes of the GRIDS instrument will then measure all three components of the ion drift velocity, in addition to the ion temperature, density, and light/heavy composition ratio.

It is worth noting that any measurement by an RPA or a drift meter can be adversely affected by electronic noise, grid geometry, unknown variations in the spacecraft potential, and variations in the sheath dimensions, all of which can introduce biases to parameters inferred from the measurements. These effects have been well-studied by a host of other authors, including Chao et al., ${ }^{15}$ Klenzing et al., ${ }^{11}$ Debchoudhury and Earle, ${ }^{16}$ and references contained therein. In particular, the Debchoudhury reference provides quantitative simulation results in a format that can be scaled to instruments having different form factors; it is a particularly valuable reference for quantitatively assessing the impact of various noise effects on measurements inferred from instruments such as the GRIDS device.

\section{CONCLUSIONS}

This paper describes the design of the GRIDS instrument for the petitSat mission. The functionality of the instrument has been detailed and validated through bench-top testing and end-to-end testing in a plasma chamber. The instrument is capable of making the required measurements for the petitSat mission but could also potentially be useful in a wide variety of science experiments requiring planetary ionospheric plasma measurements.

\section{ACKNOWLEDGMENTS}

The work presented in this paper was supported by NASA (Grant Nos. 80NSSC18K0914 and 80NSSC18K0925).

\section{DATA AVAILABILITY}

The data that support the findings of this study are available from the corresponding author upon reasonable request.

\section{REFERENCES}

${ }^{1}$ E. S. Miller, H. Kil, J. J. Makela, R. A. Heelis, E. R. Talaat, and A. Gross, “Topside signature of medium-scale traveling ionospheric disturbances," Ann. Geophys. 32(8), 959-965 (2014).

${ }^{2}$ L. Fanelli, S. Noel, G. D. Earle, C. Fish, R. L. Davidson, R. V. Robertson, P. quis, V. Garg, N. Somasundaram, L. Kordella, and P. Kennedy, "A versatile retarding potential analyzer for nano-satellite platforms," Rev. Sci. Instrum. 86, 124501 (2015).

${ }^{3}$ W. B. Hanson, R. A. Heelis, R. A. Power, C. R. Lippincott, D. R. Zuccaro, B. J. Holt, L. H. Harmon, and S. Sanatani, Space Sci. Instrum. 5, 503 (1981).

${ }^{4}$ H. Kil and R. A. Heelis, J. Geophys. Res. 103, 407, https://doi.org/10.1029/ 97ja02698 (1998).

${ }^{5}$ F. J. Rich, User's Guide for the Topside Ionospheric Plasma Monitor (SSIES, SSIES2, and SSIES-3) on Spacecraft of the Defense Meterological Satellite Program (Air Force Phillips Laboratory, Hanscome AFB, MA, 1994), p. 25.

${ }^{6}$ R. A. Heelis and W. B. Hanson, "Measurements of thermal ion drift velocity and temperature using planar sensors," in Measurement Techniques in Space Plasmas: Particles, edited by R. F. Pfaff, J. Borovsky, and D. T. Young (American Geophysical Union, 2013).

${ }^{7}$ R. A. Heelis, R. A. Stoneback, M. D. Perdue et al., Space Sci. Rev. 212, 615 (2017).

${ }^{8}$ E. C. Whipple, Proc. IRE 47, 2023 (1959).

${ }^{9}$ W. C. Knudsen, J. Geophys. Res. 71, 4669, https://doi.org/10.1029/JZ071i019 p04669 (1966).

${ }^{10}$ D. R. Zuccaro and B. J. Holt, J. Geophys. Res. 87, 8327, https://doi.org/10.1029/ JA087iA10p08327 (1982).

${ }^{11}$ J. H. Klenzing, G. D. Earle, and R. A. Heelis, "Errors in ram velocity and temperature measurements inferred from satellite-borne retarding potential analyzers," Phys. Plasmas 15, 062905 (2008).

${ }^{12}$ C. K. Chao and S.-Y. Su, Phys. Plasmas 7, 101 (2000).

${ }^{13}$ E. Robertson, G. Earle, and J. Green, “A configurable ion source for validating spaceflight-based thermal plasma measurement systems,” Rev. Sci. Instrum. 90(9), 093302 (2019).

${ }^{14}$ E. F. Robertson, "Validation and characterization of a laboratory ion source for testing thermal space-plasma instruments," Ph.D. dissertation (Virginia Tech, 2019).

${ }^{15}$ C. K. Chao, S.-Y. Su, and H. C. Yeh, "Grid effects on the derived ion temperature and ram velocity from the simulated results of the retarding potential analyzer data," Adv. Space Res. 32, 2361 (2003).

${ }^{16}$ S. Debchoudhury and G. Earle, "Noise-induced errors in geophysical parameter estimation from retarding potential analyzers in low Earth orbit," Phys. Plasmas 24, 042902 (2017). 